\title{
Dark state atoms trapping in a magic-wavelength optical lattice near the nanofiber surface
}

\author{
Dianqiang Su (苏殿强 $)^{1,2}$ ，Xiateng Qin (秦夏腾) ${ }^{1,2}$ ，Yuan Jiang (蒋 源 $)^{1,2}$ ，Kaidi Jin (靳凯迪 $)^{1,2}$ ，Zhonghua Ji (姬中华 $)^{1,2}$ ， \\ Yanting Zhao (赵延霆) $)^{1,2^{*}}$, Liantuan Xiao (肖连团) $)^{1,2}$, and Suotang Jia (贾锁堂) $)^{1,2}$ \\ ${ }^{1}$ State Key Laboratory of Quantum Optics and Quantum Optics Devices, Institute of Laser Spectroscopy, Shanxi University, Taiyuan 030006, China \\ ${ }^{2}$ Collaborative Innovation Center of Extreme Optics, Shanxi University, Taiyuan 030006, China
}

*Corresponding author: zhaoyt@sxu.edu.cn

Received August 1, 2021 | Accepted November 2, 2021 | Posted Online November 19, 2021

\begin{abstract}
We report the experimental realization of dark state atoms trapping in a nanofiber optical lattice. By applying the magicwavelength trapping potentials of cesium atoms, the AC Stark shifts are strongly suppressed. The dark magneto-optical trap efficiently transfers the cold atoms from bright $\left(6 S_{1 / 2}, F=4\right)$ into dark state $\left(6 S_{1 / 2}, F=3\right)$ for hyperfine energy levels of cesium atoms. The observed transfer efficiency is as high as $98 \%$ via saturation measurement. The trapping lifetime of dark state atoms trapped by a nanofiber optical lattice is also investigated, which is the key element for realizing optical storage. This work contributes to the manipulation of atomic electric dipole spin waves and quantum information storage for fiber networks.
\end{abstract}

Keywords: nanofiber; atomic trapping; optical lattice; dark state.

DOI: 10.3788/COL202220.020201

\section{Introduction}

Along with the development of the magneto-optical trap (MOT) technique, many species of atoms have been successfully trapped and greatly isolated from the external environment ${ }^{[1-3]}$. It makes them promising for the realizations of quantum repeater and quantum networks ${ }^{[4-6]}$. In order to realize the control and manipulation for atomic states, the trapped atoms need to efficiently interface with resonant probe light via atom-light interaction $^{[7-11]}$. In this respect, a tapered optical nanofiber provides an ideal platform. The strong confinement evanescent field near the nanofiber improves atom-light coupling and allows atomic trapping near the nanofiber surface ${ }^{[12-14]}$. In recent years, twocolor trappings including a state-insensitive nanofiber trap for atoms have been realized ${ }^{[15-17]}$. The atoms are trapped in a one-dimensional optical lattice near the nanofiber surface. Due to the collisional blockade effect ${ }^{[18,19]}$, at most, one atom can be located for each optical lattice site. The strong coupling between the trapped atomic array and fiber-guided mode contributes to the study of chiral quantum optics ${ }^{[20]}$, collective excitation $^{[21]}$, and correlating photons under nonlinear response ${ }^{[22]}$. Using electromagnetically induced transparency, slow light and the storage of optical pulses are also realized in the nanofiber optical lattice ${ }^{[23,24]}$. In the nanofiber trapping regime, the optical depth (OD) can reach a few percent per atom, which enables the large OD with a trapped atomic array and confirms it as a viable platform for studying atom-light interactions.
In this Letter, we focus on the optical nanofiber trapping scheme. By applying dark MOT (DMOT) technique, the trapped cesium atoms are transferred to the lower hyperfine ground state (dark state). The minimized atomic collisions and radiation pressure allow the higher atomic density and lower atomic temperature, which are the critical elements to the loading efficiency of the nanofiber optical lattice. With the application of DMOT, the bright-dark state transfer efficiency of nanofiber trapped atoms reaches $98 \%$, providing the essential requirements for developing the quantum repeater with a fiberbased hybrid system ${ }^{[21]}$.

\section{Experiments}

As shown in Fig. 1(a), the diameter of the nanofiber is $500 \mathrm{~nm}$ over a length of $5 \mathrm{~mm}$ fabricated from a single mode fiber (Fibercore, SM800-5.6-125) by flame brushing ${ }^{[25-28]}$. The designed diameter guarantees the quasi-fundamental mode $\mathrm{HE}_{11}$ along the nanofiber, and the designed taper angle (2 mrad) guarantees the adiabatical transformations for fiber-guided modes from both sides. Owing to the high transmission (99.5\%), the nanofiber can tolerate power of input light over $30 \mathrm{~mW}$ in a vacuum. Figure 1(b) shows the schematic of the experimental setup. A couple of counter-propagating red-detuned lights (935 nm, $0.35 \mathrm{~mW}$ for each beam) create the attractive potential. Another couple of parallel polarized blue-detuned lights 


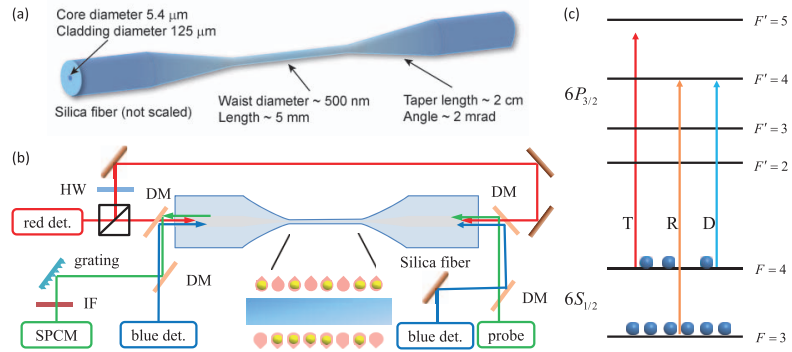

Fig. 1. (a) Schematic of a nanofiber with $500 \mathrm{~nm}$ in diameter. (b) Schematic of the apparatus. The atomic arrays are trapped in the evanescent field near the nanofiber surface. The magic-wavelength optical lattice consists of two pairs of counter-propagating beams, including red detuning standing wave light $(935 \mathrm{~nm})$ to make the attractive potential and blue detuning traveling wave light $(686.1 \mathrm{~nm}, 686.5 \mathrm{~nm})$ to make the repulsive penitential. DM, dichroic mirror; HW, half-waveplate; SPCM, single photon counter module. (c) Energy level diagram of cesium. $T$, trapping light; $R$, repumping light; $D$, depumping light.

(686.1 nm, $686.5 \mathrm{~nm}, 5 \mathrm{~mW}$ for each beam) create repulsive potential. Overlapping the van der Waals potential from the nanofiber surface, the evanescent field of trapping lights constructs the nanofiber optical lattice. The application of the magic wavelength for trapping lights strongly immunizes the AC Stark shifts of cesium energy levels. The polarization of fiber-guided lights is calibrated by the Rayleigh scattering induced by the imperfection of nanofibers. With theoretical calculation, the center of the optical lattice trap is located $173 \mathrm{~nm}$ away from the nanofiber surface. The trapping frequencies are about $352 \mathrm{kHz}$ radially, $77 \mathrm{kHz}$ azimuthally, and $500 \mathrm{kHz}$ along the nanofiber. The probe light is coupled into the nanofiber from one side and detected with the single photon counter module (SPCM), filtering via volume Bragg gratings (VBGs, OptiGrate, SPC-852) and interference filter (Semrock, LL01852-12.5). Figure 1(c) shows the hyperfine energy level of cesium atoms used in our experiment. The trapping frequency is reddetuned $12 \mathrm{MHz}$ from the $6 S_{1 / 2}, F=4 \rightarrow 6 P_{3 / 2}, F^{\prime}=5$ transition, and the repumping light is resonant on $6 S_{1 / 2}, F=$ $3 \rightarrow 6 P_{3 / 2}, F^{\prime}=4$. Here, the center region of the repumping beam is dumped and substituted by the depumping light, for which the diameter is $3 \mathrm{~mm}$, overlapping the trapped atomic ensemble. The corresponding intensity of the depumping light is about $0.3 \mathrm{~mW} / \mathrm{cm}^{2}$, and the frequency is resonant on the $6 S_{1 / 2}, F=4 \rightarrow 6 P_{3 / 2}, F^{\prime}=4$ transition.

\section{Results}

To increase the loading efficiency for the nanofiber optical lattice, we optimized the polarization gradient cooling progress and measured the corresponding atomic temperature from DMOT. The trapping power of each beam is fixed at $4.7 \mathrm{~mW} / \mathrm{cm}^{2}$. After $1.5 \mathrm{~s}$, the loaded atomic number from normal MOT becomes saturated. In the polarization gradient cooling stage, the trapping frequency is detuned from $-12 \mathrm{MHz}$ to $-60 \mathrm{MHz}$ within $5 \mathrm{~ms}$.
The powers of trapping light and repumping light are reduced to zero. Meanwhile, the depumping light is always turned on.

By applying time of flight, we measured the temperature of nanofiber trapped atoms loading from DMOT, which is compared with normal MOT, as shown in Fig. 2. The changes of the Gaussian diameter of the atomic ensemble obey

$$
\sigma_{x, y}=\sqrt{\sigma_{x, y}^{2}(0)+\frac{k_{B} T_{x, y}}{m} t^{2}},
$$

where $m$ is the atomic mass, $T_{x, y}$ is the temperature of the atomic ensemble considering the weighting coefficients in the $x$ and $y$ directions, $k_{B}$ is the Boltzmann constant, and $t$ is the atomic flying time. In the imaging progress, the duration of the probe pulse is $1 \mathrm{~ms}$, which is resonant on $6 S_{1 / 2}, F=3 \rightarrow 6 P_{3 / 2}, F^{\prime}=2$ for DMOT atoms $\left(6 S_{1 / 2}, F=4 \rightarrow 6 P_{3 / 2}, F^{\prime}=5\right.$ for normal MOT atoms). An imaging size calibrated camera records the corresponding atomic ensemble with different atomic flying times. According to Eq. (1) and the measured atomic size from Fig. 2, the calculated atomic temperatures for normal MOT and DMOT are separately $65.8 \mu \mathrm{K}$ and $44.8 \mu \mathrm{K}$, proving the lower atomic temperature for DMOT.

Figure 3 shows the absorption spectra of nanofiber trapped atoms loading from DMOT and normal MOT. After the polarization gradient cooling progress, a frequency fixed probe pulse duration of $1 \mathrm{~ms}$ is input into the nanofiber, interacting with the nanofiber trapped atoms by the evanescent field of the fiberguided mode. The probe power is set to be $1 \mathrm{pW}$ to avoid the recoil heating effect ${ }^{[29]}$. For resonant transmission spectra, the OD can be derived from a simple model:

$$
T(w)=\exp \left[-\mathrm{OD} \frac{1}{1+4\left(w-w_{0}\right)^{2} / \Gamma^{2}}\right],
$$
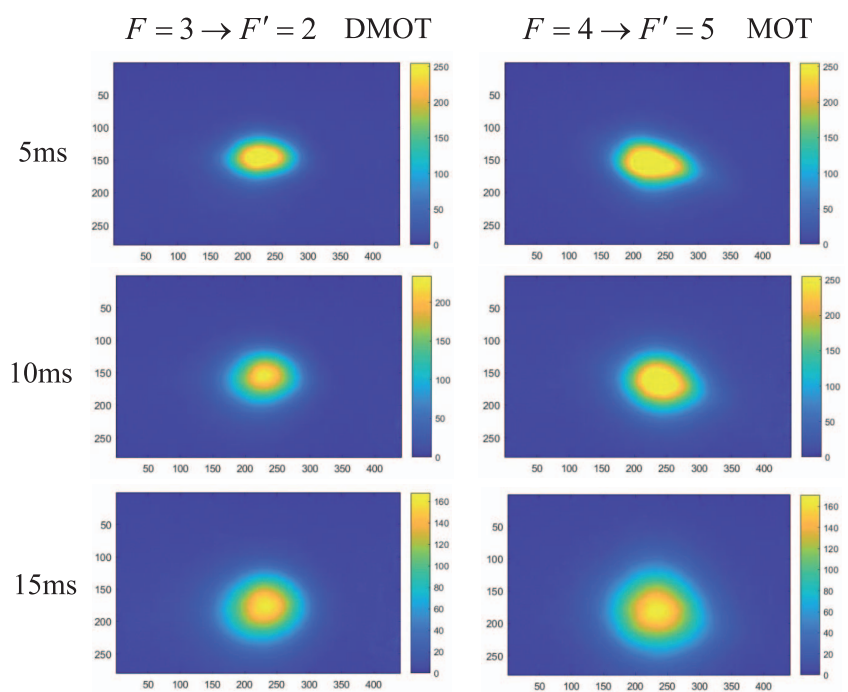

Fig. 2. Temperature measurement of atoms trapped from DMOT and normal MOT. After turning off the magnetic field, the atoms are probed with different flying times. 

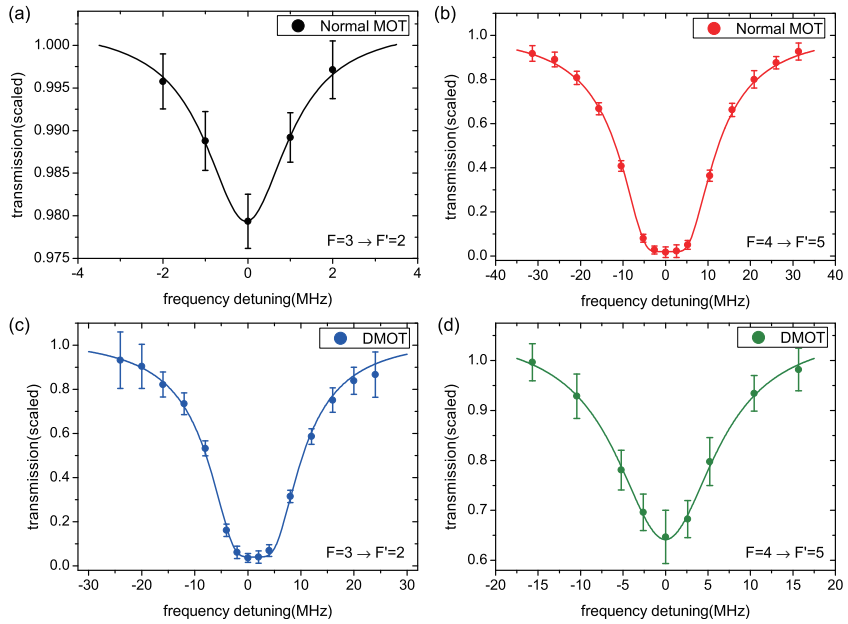

Fig. 3. (a), (b) Absorption of nanofiber trapped atoms loading from normal MOT. The corresponding frequency transitions are separately $6 S_{1 / 2}, F=3 \rightarrow 6 P_{3 / 2}$, $F^{\prime}=2$ and $6 S_{1 / 2}, F=4 \rightarrow 6 P_{3 / 2}, F^{\prime}=5$. (c), (d) Absorption of trapped atoms loading from DMOT with the same transitions as in (a) and (b). Each dot is averaged from 10 experimental runs with the error bar representing the standard errors in photon statistics. The solid lines are the fitted consequence in theory.

where $w_{0}$ is the resonant frequency, and $\Gamma$ is the full width at half-maximum (FWHM) of the spectra. Figures 3(a) and 3(b) show the absorption spectra of nanofiber trapped atoms loading from normal MOT. According to Eq. (2), the fitted OD is separately $0.02 \pm 0.002$ and $14.4 \pm 0.2$, corresponding to the transmission $6 S_{1 / 2}, F=3 \rightarrow 6 P_{3 / 2}, F^{\prime}=2$ and $6 S_{1 / 2}, F=4 \rightarrow$ $6 P_{3 / 2}, F^{\prime}=5$. The large OD of the bright state indicates that nearly all trapped atoms are populated into the higher hyperfine level $6 S_{1 / 2}, F=4$ for the normal MOT. Figures 3(c) and 3(d) show the absorption spectra of trapped atoms loading from DMOT. The fitted OD is $9.3 \pm 0.2$ and $0.55 \pm 0.02$, corresponding to the same transmissions as in Figs. 3(a) and 3(b). The increased OD for the $6 S_{1 / 2}, F=3 \rightarrow 6 P_{3 / 2}, F^{\prime}=2$ transition interprets that most trapped bright atoms have been transferred to the dark state with DMOT. For fitting consequences of trapped atoms with normal MOT (red line) and DMOT (blue line), the frequency shifts are $0.33 \pm 0.07 \mathrm{MHz}$ and $1.24 \pm 0.08 \mathrm{MHz}$, benefitting from the application of cesium magic-wavelength lights for trapping potential. The AC Stark shift effect and inhomogeneous Zeeman broadening are strongly eliminated. Compared with Figs. 3(a) and 3(d), the larger frequency shifts of Figs. 3(b) and 3(c) are induced by the perturbations from atom-atom interactions in the optical lattice. These coherent dipole-dipole couplings are related to the lattice geometries and OD of trapped atoms ${ }^{[30]}$, which could be ignored when the OD is very small.

To quantify the trapped atomic numbers and bright-dark state transfer efficiency, we measured the absorption power for nanofiber trapped atoms loading from DMOT, as shown in Fig. 4. The black and red lines are fitted based on the generalized Beer's law. In the limit of large input power and saturated absorption, we can conclude that (a)

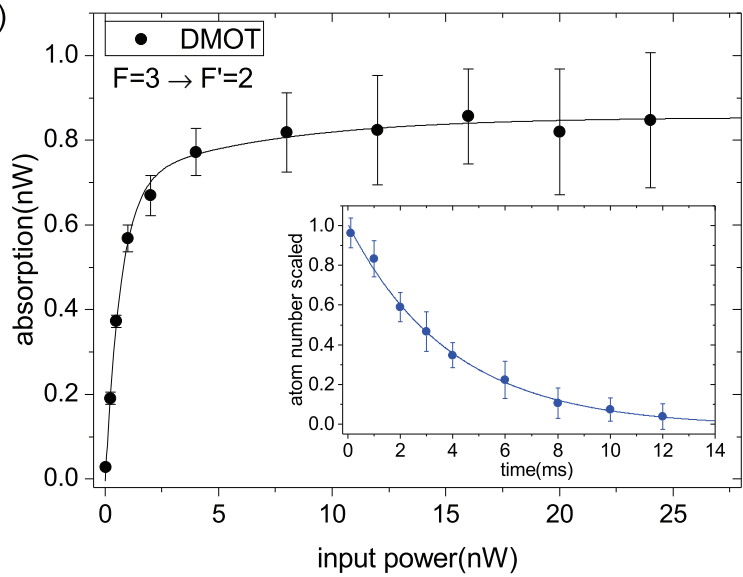

(b)

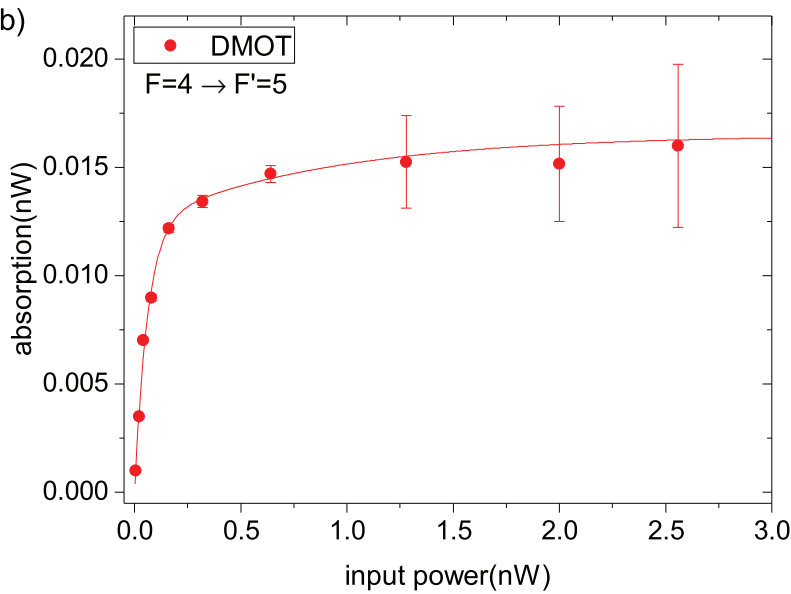

Fig. 4. Saturation measurement of power absorption for nanofiber trapped atoms with DMOT. (a) For transition $6 S_{1 / 2}, F=3 \rightarrow 6 P_{3 / 2}, F^{\prime}=2$. The inset shows the lifetime of nanofiber trapped atoms in the optical lattice with exponential fitting (blue line). (b) For transition $6 S_{1 / 2}, F=4 \rightarrow 6 P_{3 / 2}, F^{\prime}=5$. The black and red lines are fitted results following a generalized Beer's law. The data are averaged 10 times with a standard error bar.

$$
N \approx \frac{P_{\mathrm{abs}}}{P_{\mathrm{sat}}}
$$

where $P_{\text {sat }}=3.8 \mathrm{pW}$ is the radiated power of a single saturated cesium atom ${ }^{[16]}$. Based on Eq. (3) and saturated power fitted from the generalized Beer's law, we can infer that the trapped atomic numbers populated into states $F=3$ and $F=4$ are $232 \pm 26$ and $4.2 \pm 0.8$. It indicates that approximately $98 \%$ of nanofiber trapped atoms have been transferred to dark state $F=3$. The OD per atom is $4 \%$, as concluded from the confirmed atomic number and OD.

The lifetime of trapped atoms in the nanofiber optical lattice is also measured, as shown in the inset of Fig. 4(a). The input power is fixed at a saturation level $(8 \mathrm{nW})$ to meet the premise of Eq. (3). For lifetime measurement of the inset of Fig. 4(a), the atomic number is calculated and scaled. For nanofiber trapped atoms with a dark state, the ultimate exponential fitted lifetime is $3.9 \pm 0.2 \mathrm{~ms}$ (blue line). A further temperature cooling for the trapped atoms is needed to increase the lifetime in future work. 


\section{Conclusion}

In conclusion, we have realized dark state atomic trapping in a nanofiber optical lattice by applying the DMOT. The brightdark state transfer efficiency of trapped atoms reaches about $98 \%$, confirmed by the saturation measurement of power absorption. The AC Stark shift is also strongly suppressed by using the cesium magic-wavelength trapping potential (bluedetuned traveling wave and red-detuned standing wave). This work contributes to the realization of a fiber-based quantum repeater and hybrid quantum network construction.

\section{Acknowledgement}

This work was supported by the National Key Research and Development Program of China (No. 2017YFA0304203), National Natural Science Foundation of China (Nos. 6210031464, 61875110, 12034012, and 12074231), NSFC Project for Excellent Research Team (No. 61121064), "1331 KSC", PCSIRT (No. IRT_17R70), National Time Service Center (NTSC) of the Chinese Academy of Sciences (CAS) (No. 2009DP173082), State Key Laboratory of Surface Physics, Fudan University (No. KF2020_01), and 111 Project (No. D18001).

\section{References}

1. E. L. Raab, M. Prentiss, A. Cable, S. Chu, and D. E. Pritchard, "Trapping of neutral sodium atoms with radiation pressure," Phys. Rev. Lett. 59, 2631 (1987).

2. G. Colzi, G. Durastante, E. Fava, S. Serafini, G. Lamporesi, and G. Ferrari, "Sub-Doppler cooling of sodium atoms in gray molasses," Phys. Rev. A 93, 023421 (2016).

3. D. Nath, R. K. Easwaran, G. Rajalakshmi, and C. S. Unnikrishnan, "Quantum-interference-enhanced deep sub-Doppler cooling of $39 \mathrm{~K}$ atoms in gray molasses," Phys. Rev. A 88, 053407 (2013).

4. H. J. Kimble, "The quantum internet," Nature 453, 1023 (2008).

5. Z. S. Yuan, Y. A. Chen, B. Zhao, S. Chen, J. Schmiedmayer, and J. W. Pan, "Experimental demonstration of a BDCZ quantum repeater node," Nature 454, 1098 (2008).

6. B. Zhao, Y. A. Chen, X. H. Bao, T. Strassel, C. S. Chuu, X. M. Jin, J. Schmiedmayer, Z. S. Yuan, S. Chen, and J. W. Pan, "A millisecond quantum memory for scalable quantum networks," Nat. Phys. 5, 95 (2009).

7. C. W. Chou, H. de Riedmatten, D. Felinto, S. V. Polyakov, S. J. van Enk, and H. J. Kimble, "Measurement-induced entanglement for excitation stored in remote atomic ensembles," Nature 438, 828 (2005).

8. C. W. Chou, J. Laurat, H. Deng, K. S. Choi, H. de Riedmatten, D. Felinto, and H. J. Kimble, "Functional quantum nodes for entanglement distribution over scalable quantum networks," Science 316, 1316 (2007).

9. N. Sangouard, C. Simon, H. de Riedmatten, and N. Gisin, "Quantum repeaters based on atomic ensembles and linear optics," Rev. Mod. Phys. 83, 33 (2011).

10. E. Ghasemian and M. K. Tavassoly, "Population dynamics of ultra-cold atoms interacting with radiation fields in the presence of inter-atomic collisions," Chin. Opt. Lett. 19, 122701 (2021).
11. W. Zhuang, Y. Zhao, S. K. Wang, Z. J. Fang, F. Fang, and T. C. Li, "Ultranarrow bandwidth Faraday atomic filter approaching natural linewidth based on cold atoms," Chin. Opt. Lett. 19, 030201 (2021).

12. D. Q. Su, R. J. Liu, Z. H. Ji, X. D. Qi, Z. X. Song, Y. T. Zhao, L. T. Xiao, and S. T. Jia, "Observation of ladder-type electromagnetically induced transparency with atomic optical lattices near a nanofiber," New J. Phys. 21, 043053 (2019).

13. Z.X. Song, X. Y. Yue, Y. Luo, H. D. Li, and Y. T. Zhao, "Absorption saturation measurement using the tapered optical nanofiber in a hot cesium vapor," Chin. Opt. Lett. 17, 031901 (2019).

14. D. Q. Su, R. J. Liu, C. B. Zhang, Z. H. Ji, Y. T. Zhao, L. T. Xiao, and S. T. Jia, "Laser frequency stabilization in sub-nanowatt level using nanofibers," Phys. D 51, 465001 (2018).

15. E. Vetsch, D. Reitz, G. Sagué, R. Schmidt, S. T. Dawkins, and A. Rauschenbeutel, "Optical interface created by laser-cooled atoms trapped in the evanescent field surrounding an optical nanofiber," Phys. Rev. Lett. 104, 203603 (2010).

16. S. T. Dawkins, R. Mitsch, D. Reitz, E. Vetsch, and A. Rauschenbeutel, "Dispersive optical interface based on nanofiber-trapped atoms," Phys. Rev. Lett. 107, 243601 (2011).

17. A. Goban, K. S. Choi, D. J. Alton, D. Ding, C. Lacroŭte, M. Pototschnig, T. Thiele, N. P. Stern, and H. J. Kimble, "Demonstration of a state-insensitive, compensated nanofiber trap,” Phys. Rev. Lett. 109, 033603 (2012).

18. T. Grünzweig, A. Hilliard, M. McGovern, and M. F. Andersen, "Neardeterministic preparation of a single atom in an optical microtrap," Nat. Phys. 6, 951 (2010).

19. A. Carpentier, Y. H. Fung, P. Sompet, A. J. Hilliard, T. G. Walker, and M. F. Andersen, "Preparation of a single atom in an optical microtrap," Laser Phys. Lett. 10, 125501 (2013).

20. P. Lodahl, S. Mahmoodian, S. Stobbe, A. Rauschenbeutel, P. Schneeweiss, J. Volz, H. Pichler, and P. Zoller, "Chiral quantum optics," Nature 541, 473 (2017).

21. N. V. Corzo, J. Raskop, A. Chandra, A. S. Sheremet, B. Gouraud, and J. Laurat, "Waveguide-coupled single collective excitation of atomic arrays," Nature 566, 359 (2019).

22. A. S. Prasad, J. Hinney, S. Mahmoodian, K. Hammerer, S. Rind, P. Schneeweiss, A. S. Sø rensen, J. Volz, and A. Rauschenbeutel, "Correlating photons using the collective nonlinear response of atoms weakly coupled to an optical mode," Nat. Photon. 14, 719 (2020).

23. C. Sayrin, C. Clausen, B. Albrecht, P. Schneeweiss, and A. Rauschenbeutel, "Storage of fiber-guided light in a nanofiber-trapped ensemble of cold atoms," Optica 2, 353 (2015).

24. B. Gouraud, D. Maxein, A. Nicolas, O. Morin, and J. Laurat, "Demonstration of a memory for tightly guided light in an optical nanofiber," Phy. Rev. Lett. 114, 180503 (2015).

25. F. Orucevi, V. Lefèvre-Seguin, and J. Hare, "Transmittance and near-field characterization of sub-wavelength tapered optical fibers," Opt. Express 15, 13624 (2007).

26. A. Stiebeiner, R. Garcia-Fernandez, and A. Rauschenbeutel, "Design and optimization of broadband tapered optical fibers with a nanofiber waist," Opt. Express 18, 22677 (2010).

27. T. Aoki, "Fabrication of ultralow-loss tapered optical fibers and microtoroidal resonators," Jpn J. Appl. Phys. 49, 118001 (2010).

28. J. E. Hoffman, S. Ravets, J. A. Grover, P. Solano, P. R. Kordell, J. D. WongCampos, L. A. Orozco, and S. L. Rolston, "Ultrahigh transmission optical nanofibers," AIP Adv. 4, 067124 (2014).

29. S. Wolf, S. J. Oliver, and D. S. Weiss, "Suppression of recoil heating by an optical lattice," Phys. Rev. Lett. 85, 4249 (2000).

30. D. E. Chang, J. Ye, and M. D. Lukin, "Controlling dipole-dipole frequency shifts in a lattice-based optical atomic clock," Phys. Rev. A 69, 023810 (2004). 